American Journal of Neuroscience 2 (1): 35-47, 2011

ISSN 1948-9900

(C) 2011 Science Publications

\title{
Dementia in Parkinson's Disease: A Clinical Review
}

\author{
${ }^{1}$ Renata Teles Vieira, ${ }^{1}$ Leonardo Caixeta, \\ ${ }^{2,3}$ Sergio Machado and ${ }^{1}$ Marcelo Caixeta \\ ${ }^{1}$ Dementia Outpatient Unit, Hospital of the Clinics, \\ Federal University of Goias (UFG), Brazil \\ ${ }^{2}$ Institute of Psychiatry, Panic and Respiration, \\ Federal University of Rio de Janeiro, Brazil \\ ${ }^{3}$ Institute of Psychiatry, Brain Mapping and Sensory Motor Integration, \\ Federal University of Rio de Janeiro, Brazil
}

\begin{abstract}
Problem statement: Idiopathic Parkinson's Disease (PD) has being increasingly seen as a neuropsychiatric entity and no longer purely neurological, given the frequency and exuberance with which the psychiatric manifestations occurs. Approach: This review will focus on the epidemiology, clinical aspects, differential diagnosis with Alzheimer's Disease (AD) associated with parkinsonism, Dementia with Lewy Bodies (LBD), Progressive Supranuclear Palsy (PSP), Corticobasal Degeneration (CBD), Multiple System Atrophy (MSA), Vascular Parkinsonism and normal pressure hydrocephalus (NPN), underlying mechanisms and treatment of dementia in PD. A literature review of the studies that investigated the dementia in PD was performed. Results: The dementia in PD has being increasingly recognized as a distinct nosological entity. Although specific etiologies for cognitive impairment remain unknown, a symptomatic treatment has been approved and strategies for early intervention are envisioned. Conclusion: Larger scale, placebo controlled clinical trials are needed to be explored in future studies to provide an evidence base to guide the management in dementia associated with PD.
\end{abstract}

Key words: Corticobasal degeneration, dementia with lewy bodies, progressive supranuclear palsy, multiple system atrophy, Parkinson's disease, vascular parkinsonism, therapeutic strategies, basal ganglia, clinical picture

\section{INTRODUCTION}

Neuropsychiatry has made progressive approaches to diseases of the central nervous system which lead to movement disorders, either by frequent changes of behavior in these entities (both in the form of comorbidities as in the form of changes secondary to the pathological substrate in the basal ganglia), or the potential for pathophysiological explanations that can be extrapolated from these basal ganglia disturbances to psychiatric disorders (for example, disorders of the obsessive-compulsive spectrum) (Lyketsos et al., 2007).

Idiopathic Parkinson's Disease (PD) has being increasingly seen as a neuropsychiatric entity and no longer purely neurological, given the frequency and exuberance with which the psychiatric manifestations occurs, making more complex the clinical picture and involving more differentiated therapeutic strategies (Lyketsos et al., 2007). PD is traditionally linked to an essentially motor disorder. The cognitive symptoms in PD were classically neglected since its original description by James Parkinson, from which an erroneous culture was established in which cognitive functions remained unchanged in the course of the disease. In the late nineteenth century Charcot already anticipated, however, that there is also cognitive impairment in PD (Teive et al., 2001; Lanska, 2010).

Nowadays it is already well established that cognitive impairment and in particular dementia in PD are more frequent with aging, which, incidentally, can be observed in general in other forms of dementia. The dementia associated with PD (PDD) has been widely recognized, justified in part by better understanding of dementia and therefore the most frequent diagnosis of this syndrome, but also because of increased survival of patients with PD (Poewe and Wenning, 2006; Gelb et al., 1999).

Corresponding Author: Leonardo Caixeta, Dementia Outpatient Unit, Hospital of the Clinics, Federal University of Goias, Brazil 
The concept of subcortical dementia associated with bradyphrenia and cognitive rigidity in PD is known. For cognition in parkinsonian disorders, this notion developed into the concept of mild cognitive impairment and fronto-executive dysfunction in particular, driven mainly by dopaminergic dysmodulation and manifesting as deficits in flexibility, planning, working memory and reinforcement learning. However, patients with Parkinson's disease could also develop a syndrome of dementia that might depend on non-dopaminergic, cholinergic cortical dysfunction (Kehagia et al., 2010).

A wide variety of cognitive impairments have been reported, even early in the course of the disease, including memory, visuospatial function and executive function. Mild cognitive impairment was found already at the time of diagnosis in some patients in an incident cohort of PD. There is some evidence that dementia is merely a progression of this early cognitive impairment, while other evidence suggests a different profile in dementia with PD and non-demented PD patients (Emre et al., 2007).

The most common definition for PDD has been the presence of PD plus the DSM-IV criteria for dementia. This criterion involves abnormalities in memory and one other domain of cognition,functional decline related to cognitive deficit(s) and preservation of consciousness. Recently, the Movement Disorders Society (MDS) task force defined core criteria for PDD as dementia syndrome having impairment in two or more cognitive domains out of attention, executive, visuospatial function and memory (Martinez-Martin et al., 2011). Mostly, Possible PDD occurs when the neuropsychological profile in one or more of the cognitive domains is considered atypical (Kovari et al., 2003).

The point prevalence of dementia in PD is close to $30 \%$ and the incidence rate is increased 4-6 times as compared to controls. The cumulative prevalence is very high, at least $75 \%$ of PD patients who survive for more than 10 years will develop dementia. The mean time from onset of PD to dementia is approximately 10 years. However, there are considerable variations. The most established risk factors for early dementia are old age, severity of motor symptoms, in particular postural and gait disturbances, mild cognitive impairment and visual hallucinations (Aarsland and Kurz, 2010).

The dementia in PD has being increasingly recognized as a distinct nosological entity. Because of the confusion that surrounds this diagnosis, began the need to propose operational criteria for the common form of dementia associated with PD (Emre et al., 2007), whose profile has been repeatedly demonstrated as different from Alzheimer's Disease (AD), but with overlap with dementia with Lewy Bodies (LBD) and the large group of parkinsonism plus. These criteria require confirmation in prospective studies of clinicalpathological correlation, in which the profile of dementia is described in detail and correlated with all potential types of pathology. The current evidence suggests that dementia in PD is better correlated with Lewy bodies in certain limbic and cortical areas (Emre, 2004; Shoulson, 1998).

This review will focus on the epidemiology, clinical aspects, differential diagnosis with Alzheimer's Disease (AD) associated with parkinsonism, Dementia with Lewy Bodies (LBD), Progressive Supranuclear Palsy (PSP), Corticobasal Degeneration (CBD), Multiple System Atrophy (MSA), Vascular Parkinsonism and Normal Pressure Hydrocephalus (NPN), underlying mechanisms and treatment of dementia in PD.

Epidemiology: The incidence of dementia in PD is six times higher than in the general population and is cumulative with ageing. In this regard, when with 85 years, the patient with PD will develop dementia in $65 \%$ of cases (this chance seems to be independent of the increasing possibility of developing dementia with ageing observed in the general population) (Emre, 2003a).

The prevalence of dementia among the holders of PD is not yet well defined, with very different estimates, ranging from $10-50 \%$. The more widespread average prevalence, however, is estimated around $30 \%$ (Emre, 2003a). In a systematic review of 12 prevalence studies that met prespecified criteria for PD or dementia in PD including 1,767 PD patients, the proportion of PD patients with dementia was $24.5 \%$ (95\% CI 17.431.5) (Aarsland et al., 2005). As a general rule, however, the incidence and especially the prevalence of dementia in PD are still not clear and are reported on a very varied way according to the author considered. The gap and the difficulty in establishing a uniform rate between the various studies falls, in most cases, in the precariousness of the definition of dementia more commonly accepted and used, one that incorporates the concept of dementia as a cortical syndrome, which obviously not coincides with the subcortical dementia characteristic of PD (Emre, 2003a).

In one study was estimated the cumulative prevalence of dementia in patients with PD followed by eight years (Aarsland et al., 2003a). The reported cumulative prevalence of dementia was high: $78 \%$ over the eight years of follow up. This work was conducted in a group of 224 patients recruited from an epidemiological survey of PD (for this study, subjects 
with cognitive deterioration in the onset of the disease were excluded). Compared with a sample population of 3,295 people who were examined after 2 and 5 years, the prevalence of dementia in 4 years was almost three times higher in the group with PD and the prevalence in 8 years was high: $78 \%$. This study may have resulted in an unexpectedly high prevalence because perhaps the large number of patients who already had dementia at baseline $(26 \%$ of the population included in the research) and the relatively high age of these patients. This is, however, one of the few longitudinal studies that used well-defined diagnostic criteria and validated assessment tools. This study indicates that pathology of PD itself is a risk factor for dementia.

The two most important risk factors for dementia in PD are age and severity of extrapyramidal signs (Levy, 2007). Among other risk factors for the development of dementia in PD, we can mention (Emre et al., 2007):

- Advanced age

- Beginning of PD in very old age

- Cognitive functions already impaired in the initial evaluation

- Rapid progression of the disease

- Low response to levodopa, with exuberant side effects

- Early presence of hallucinations

- Akinetic-rigid forms of the disease

- Severity of motor impairment

Pathology and clinical-pathological correlations: The pathology underlying dementia in PD is still controversial. Loss of cholinergic, dopaminergic and noradrenergic innervation has been suggested as the main neurochemical deficits. The nigral pathology alone is probably not sufficient to explain the development of dementia in patients with PD (Braak et al., 2003). Although there are controversies about the site and type of pathology involved, dementia is probably associated with the spread of PD injuries to other subcortical nuclei, as well as to the limbic system and cerebral cortex. According to more recent studies, the main condition appears to be a degeneration of the type of Lewy bodies with cellular and synaptic loss in cortical and limbic structures (Kovari et al., 2003; Emre, 2003a; 2003b).

Another recent study (Colosino et al., 2003), however, suggested that the reverse may not be true, i.e., the presence of limbic or cortical Lewy bodies not always is associated with dementia in PD. Other authors (Braak et al., 2003) suggest an ascending order of PD pathological changes, which develops in some susceptible types of neurons. It is reported that the lesions initially occur in some nuclei of brain stem and anterior olfactory nucleus and, after that, less vulnerable areas of cortical and subcortical nuclei are gradually affected. Disease process in the brain stem seems to follow an ascendant course, when the last cortical involvement begins with the anterior-medial temporal mesocortex and spreads to the neocortex, starting with the pre-frontal and sensory association areas, areas that are involved in cognitive functions. This pattern of ascending pathology from the brainstem to the limbic and neocortical areas can provide a perception on why cognitive changes appear relatively late in classic PD (Perry et al., 2003).

Clinical and forms of presentation: Patients with PD can, theoretically, be affected by any and various forms of dementia, as in the rest of the population. Occurs, however, a certain pattern of dementia associated with PD which is presumably caused by the same types of pathophysiological changes that also cause motor symptoms (Emre, 2004). Dementia associated with PD is characterized by (Poewe and Wenning, 2006):

- Reduction or lack of initiative for spontaneous actions

- Failure to develop successful strategies for problem solving

- Slowing of memory processing

- Slowing of global information processing

- Impairment of visual-spatial perception

- Difficulties of conceptualization

- Difficulty in list of words generation

All the features of dementia associated with PD can be viewed in Table 1. The diagnostic criteria for dementia in PD have been identified recently and divided into probable and possible criteria for dementia in PD (Table 2) (Emre et al., 2007).

Dementia associated with PD, at diagnosis, is usually mild to moderate and in clinical evaluation are more salient: slowing of psychomotor and global cognitive processing, executive dysfunction (loss in abstraction, in the formation of concepts, in the spontaneous word generation), dysfunction of the constructive and visual-spatial tasks, low yields in some mathematical tasks and secondary impairment of memory, in which the storage of information is relatively well preserved (Emre, 2004; Shoulson, 1998). 
Am. J. Neuroscience 2(1): 35-47, 2011

Table 1: Features of dementia associated with Parkinson's disease

I. Core features

Diagnosis of Parkinson's disease according to Queen Square Brain Bank criteria

2. A dementia syndrome with insidious onset and slow progression, developing within the context of established Parkinson's

disease and diagnosed by history, clinical and mental examination, defined as:

Impairment in more than one cognitive domain

Representing a decline from premorbid level

Deficits severe enough to impair daily life (social, occupational, or personal care), independent of the impairment ascribable to motor or autonomic symptoms

II. Associated clinical features

1. Cognitive features:

Attention: Impaired. Impairment in spontaneous and focused attention, poor performance in attentional tasks; performance may fluctuate

during the day and from day to day

Executive functions: Impaired. Impairment in tasks requiring initiation, planning, concept formation, rule finding, set shifting or set maintenance; impaired mental speed (bradyphrenia)

Visuo-spatial functions: Impaired. Impairment in tasks requiring visual-spatial orientation, perception, or construction

Memory: Impaired. Impairment in free recall of recent events or in tasks requiring learning new material, memory usually improves with cueing, recognition is usually better than free recall

Language: Core functions largely preserved. Word finding difficulties and impaired comprehension of complex sentences may be present

2. Behavioral features:

Apathy: decreased spontaneity; loss of motivation, interest and effortful behavior

Changes in personality and mood including depressive features and anxiety

Hallucinations: mostly visual, usually complex, formed visions of people, animals or objects

Delusions: usually paranoid, such as infidelity, or phantom boarder (unwelcome guests living in the home) delusions

Excessive daytime sleepiness

III. Features which do not exclude PDD, but make the diagnosis uncertain

Co-existence of any other abnormality which may by itself cause cognitive impairment, but judged not to be the cause of dementia, e.g., presence of relevant vascular disease in imaging

Time interval between the development of motor and cognitive symptoms not known

IV. Features suggesting other conditions or diseases as cause of mental impairment, which, when present make it impossible to reliably diagnose PDD

Cognitive and behavioral symptoms appearing solely in the context of other conditions such as:

Acute confusion due to

A. Systemic diseases or abnormalities

B. Drug intoxication

Major Depression according to DSM IV

Features compatible with "Probable Vascular dementia" criteria according to NINDS-AIREN (dementia in the context of cerebrovascular disease as indicated by focal signs in neurological exam such as hemiparesis, sensory deficits and evidence of relevant cerebrovascular disease by brain imaging AND a relationship between the two as indicated by the presence of one or more of the following: onset of dementia within 3

months after a recognized stroke, abrupt deterioration in cognitive functions and fluctuating, stepwise progression of cognitive deficits).

Table 2: Criteria for the diagnosis of probable and possible PDD

Probable PDD

A. Core features: Both must be present

B. Associated clinical features:

Typical profile of cognitive deficits including impairment in at least two of the four core cognitive domains (impaired attention which may fluctuate, impaired executive functions, impairment in visuo-spatial functions and impaired free recall memory which usually improves with cueing)

The presence of at least one behavioral symptom (apathy, depressed or anxious mood, hallucinations, delusions, excessive daytime sleepiness) supports the diagnosis of Probable PD-D, lack of behavioral symptoms, however, does not exclude the diagnosis

C. None of the group III features present

D. None of the group IV features present

Possible PDD

A. Core features: Both must be present

B. Associated clinical features:

Atypical profile of cognitive impairment in one or more domains, such as prominent or receptive-type (fluent) aphasia, or pure storage-failure type amnesia (memory does not improve with cueing or in recognition tasks) with preserved attention

Behavioral symptoms may or may not be present or

C. One or more of the group III features present

D. None of the group IV features present

Patients have a better performance on tasks of recognition in comparison to free recall tasks, which suggests that the storage capacity is preserved, as already mentioned, but the frontal strategy to access it is deficient (Behrmann et al., 2001). Small number of patients can present worsening of memory similar to 
that found in Alzheimer's Disease (AD). Perhaps in these cases occurs a co-existence of pathology related to $\mathrm{PD}$ and $\mathrm{AD}$, illustrating one of the nosographical difficulties of the moment and complicating our understanding of a "pure" dementia associated with PD. In general, however, the clinical presentation of dementia in PD differs from that of AD since dementia in PD better fits a pattern of subcortical dementia, while $\mathrm{AD}$ represents the prototype of cortical dementia. An example is that the presence of signs of severe cortical impairment (aphasia, agnosia, limbic amnesia) is rare in dementia associated with PD (Emre, 2004).

Changes in behavior are often found in patients with dementia and PD (Aarsland et al., 1999; 2009). Its precise origin, however, is difficult to be determined, since they may arise from a variety of causes, including: frequent use of dopaminergic and anticholinergic medications for this population of patients, presence of pathology (Lewy bodies) traditionally linked to the evocation of behavioral symptoms, tendency to present episodes of mental confusion by polymedication usually required and the intense and serious motor limitations, among others. REM sleep Behavior Disorder (RBD) is a phenomenon often seen in patients with PD and increasingly recognized in clinics. This may be even more the case for patients who eventually develop dementia. A close relationship between the pathology of synuclein and RBD was recently described, associated with a striking observation that the RBD preceded dementia or parkinsonism in an average of 10 years (ranging from 2-29). The authors of this study suggested that in the context of degenerative dementia or parkinsonism, RBD often reflects an underlying synucleopathy (Boeve et al., 2003).

It has not yet been established, without doubt, if the cognitive deficits found in PD patients without dementia are precursors of dementia. Nevertheless, it is interesting to examine such deficits, which may give rise to some clues as to the location of the underlying pathology to the cognitive deficits (Emre, 2004). Several recent studies have evaluated the different types of cognitive functions in individuals with PD and without dementia (Crucian et al., 2003; McNamara et al., 2003; Kensinger et al., 2003; Peran et al., 2003; Foltynie et al., 2004).

Differential diagnosis: The differential diagnosis of dementia in PD to other forms of dementia with parkinsonism can be extremely laborious and complex, because very often there are overlaps between the clinical pictures and neuropsychological presentations. The main differential diagnoses to be considered with PD dementia are:
- Alzheimer's Disease (AD) associated with parkinsonism

- Dementia with Lewy Bodies (LBD)

- Progressive Supranuclear Palsy (PSP)

- Corticobasal Degeneration (CBD)

- Multiple System Atrophy (MSA)

- Vascular Parkinsonism (usually associated with subcortical arteriosclerotic encephalopathy or Binswanger's disease)

- Parkinsonism linked to the normal pressure hydrocephalus

Next, it will be detailed some important aspects of the differential diagnoses listed above.

Relation of dementia in PD with Lewy Bodies Dementia (LBD): LBD is a syndrome clinically defined and consists of a primary form of dementia characterized by visual-perceptual alterations and executive dysfunction accompanied by (McKeith et al., 2005) prominent visual hallucinations (Lyketsos et al., 2007), fluctuation of attention and parkinsonism (Teive et al., 2001; Lanska, 2010). The presence of the typical cognitive profile with at least two of these three central characteristics set the diagnosis of probable LBD (McKeith et al., 2005).

PD and LBD are two common presentations of the same underlying pathological process (Lewy body disease), which is supposed to be related to deregulation of a protein from the synapse, the alphasynuclein (Burn, 2006). The relationship and differentiation between dementia in PD and LBD have been traditional cause for much dispute and confusion, although there exist some clinical, pathological and neuroimaging data that can assist in better definition of each, though not definitively, given the absence of a biological marker able to be a gold standard in the differentiation of both entities (Burn, 2006).

The misdiagnosis comes from the fact that the dementia in PD and LBD share many characteristics: cognitive profile, attentional performance, neuropsychiatric symptoms, sleep disorder, autonomic dysfunction, type and severity of parkinsonism, sensitivity to the neuroleptic agents and responsiveness to anticholinesterasics. Psychiatrically, dementia with Lewy bodies and Parkinson's disease dementia may be accompanied by depression and (mostly visual) hallucinations (McKeith et al., 2004). The age of onset, temporal course and responsiveness to levodopa therapy, however, are points of difference between the two entities (Mollenhauer et al., 2010). Yet following the clinical point of view, the postural instability and gait changes seem to be more frequent in LBD. In the same line of reasoning, but according to the 
neuropathological point of view, the deposition of beta amyloid protein is more marked and more directly related to cognitive impairment in LBD than in the PD dementia; there seems to be greater involvement of the fronto-hippocampal projections in LBD, as indicated by the grater executive dysfunction in this entity; the greater abundance of Lewy bodies on temporal cortical regions in LBD seems to associate with higher frequency of visual hallucinations and delusions; the differential pathological involvement of the striatum between the two entities can explain the specific pattern of parkinsonian features displayed in each of them (Aarsland et al., 2004).

The current guidelines suggest that for the establishment of LBD diagnosis it is necessary that the beginning of symptoms of dementia precedes or at least occurs simultaneously with the signs of parkinsonism (McKeith et al., 2005). When the period between the onset of parkinsonian symptoms and the subsequent onset of dementia is larger than one year, the most probable diagnosis is that of dementia associated with PD: this is called the "one year rule". Obviously, such conduct may seem arbitrary and lacking clinical and neuropathological rational bases (Emre et al., 2007) and has been criticized by authors who consider LBD and dementia in PD as the opposite ends of the same spectrum of disorders (McKeith, 2007). Reinforces this "spectrum theory" the fact of both entities have in common the same type of histopathological marker (Lewy bodies), although its distribution has slight differences in the brain (more conspicuous in the cortical areas in LBD, unlike the subcortical predominance in PD dementia).

Dementia in PD $\times$ AD $\times$ LBD $\times$ PSP: There are several difficulties in that differential diagnosis, especially with regard to the proximity of both in several of its features. There is strong evidence that the PD dementia and AD share some of the neuropathological (e.g., the presence of senile plaques, neurofibrilar and granulovacuolar degeneration) and neurochemical (damage to the cholinergic Nucleus Basalis of Meynert, damage to the noradrenergic pathways by the level of locus ceruleus, damage to the mesocortical dopaminergic systems, deficit of cortical nicotinic receptors) mechanisms, common between the two entities. It is worth emphasizing, however, that those PD patients with more severe dementia and in later stages who have such biochemical and pathological changes similar to AD. That said, it becomes clearer understand why some authors propose the conception of a continuum between "pure" PD and $\mathrm{AD}$, passing by the dementia associated with PD (Galpern and Lang, 2006).

Aarsland et al. (2003b) conducted an important study comparing the neuropsychological profile of dementia in four different neurodegenerative disorders: dementia in PD, dementia with Lewy bodies (LBD), $\mathrm{AD}$ and progressive supranuclear palsy (PSP). This study used the Dementia Rating Scale (a scale which is particularly sensitive to executive dysfunction) to compare the patients with different forms of dementia, finding a different profile of cognitive deficits among these different diseases. In general, the findings reinforce the similarities between PD dementia and LBD, as well as the differences they have when compared to AD. In contrast to $\mathrm{AD}$, patients with $\mathrm{PD}$ dementia and LBD had greater memory scores, but lower initiation and perseveration scores, as well as lower construction scores and LBD patients alone had lower scorers on conceptualization. Compared to patients with PSP, those with LBD and PD dementia had lower memory scores. There were no significant differences in none of the scores between the individuals with PD dementia and LBD in the group with severe dementia; the only difference was a high score of conceptualization in patients with PD dementia in comparison to patients with LBD in the group that had mild to moderate dementia. The study further emphasized the similarities between LBD and PD dementia and the differences between the cognitive profiles among these two disorders and AD. Since the memory scores of patients with PD dementia and LBD located between AD and PSP scores, authors suggest that the cognitive pattern in LBD and PD may reflect an overlap of subcortical deficits with deficits typically associated with AD. This is however difficult to accept because, as the authors admit, the Dementia Rating Scale does not discriminate the type of loss of memory, i.e., whether it is caused by deficiency of encoding or retrieval. Initial work showed that $\mathrm{AD}$ is typically associated with encoding, while PD dementia and LBD are associated with recovery deficits. The authors also concluded that, because of the almost overlapping clinical profiles, PD dementia and LBD can be seen as parts of a spectrum of disorders with Lewy bodies.

Another recent work (which also compared the clinical characteristics of PD dementia with LBD and $\mathrm{AD})$ reached conclusions similar to those outlined in the preceding paragraph (Noe et al., 2004). In this study by Noe and collaborators, patients with LBD, PD dementia and $\mathrm{AD}$ were matched by the severity of dementia. Psychosis associated with cognitive deterioration in the onset of the disease was more frequent in individuals with LBD; patients with LBD and PD dementia performed significantly worse in tasks of attention and better on tests of memory than those with AD. There were no significant differences between the group with PD dementia and patients with LBD in any of the neuropsychological tests performed. 
Am. J. Neuroscience 2(1): 35-47, 2011

Table 3: Comparison of clinical features of Lewy Body Dementia, PD dementia and Alzheimer's disease

\begin{tabular}{|c|c|c|c|}
\hline & Lewy-body dementia & PD dementia & Alzheimer's disease \\
\hline \multicolumn{4}{|l|}{ Clinical features } \\
\hline \multirow[t]{2}{*}{ Common presentation } & Psychotic symptoms and/or & Parkinsonian features & \\
\hline & Parkinsonian features. & & Memory decline \\
\hline Psychotic symptoms & Early visual hallucinations & Associated with exposure & Usually later in disease \\
\hline Memory decline & with or without delusions. & to PD pharmacotherapy & process. \\
\hline \multirow[t]{2}{*}{ Speech impairment } & $\begin{array}{l}\text { As disease progresses, } \\
\text { particularly in accessing memories. }\end{array}$ & $\begin{array}{l}\text { Difficulty accessing } \\
\text { memories }\end{array}$ & $\begin{array}{l}\text { Early global and progressive } \\
\text { difficulty in forming memories. }\end{array}$ \\
\hline & Usually late & Hypophonia, dysarthria & Aphasia, Paraphasia \\
\hline \multicolumn{4}{|l|}{ Parkisonian features } \\
\hline Tremor at rest & Present in $20-50 \%$ & Present in $75 \%$ & Only in late disease \\
\hline Rigidity & Common & Common & Only in late disease \\
\hline Gait abnormality & Early in disease & Early or late in disease & Late in disease \\
\hline \multirow{2}{*}{$\begin{array}{l}\text { Response to levodopa } \\
\text { Antipsychotic sensitivity }\end{array}$} & Variable & Common & NA \\
\hline & Can be extreme & $\begin{array}{l}\text { Variable, increased } \\
\text { parkinsonism at higher dosages }\end{array}$ & $\begin{array}{l}\text { Development of Parkinsonism } \\
\text { at higher dosages }\end{array}$ \\
\hline
\end{tabular}

Legend: NA, not applicable

The differences between patients with $\mathrm{AD}, \mathrm{LBD}$ and "PD with cognitive deterioration" were also studied by using the clock design test-CDT (Cahn-Weiner et al., 2003). There were no significant differences among the three groups in an overall quantitative measure of performance in the CDT. There were, however, qualitative differences: the LBD patients were more likely to make conceptual errors than those with $\mathrm{AD}$ or PD and patients with LBD made more planning errors than those with AD. These differences resulted in a considerable accuracy of the classification of approximately $70 \%$ between LBD versus DA and LBD versus PD with cognitive worsening. The authors concluded that, despite some differences in quality characteristics, as a single instrument, the CDT offers limited capacity to discriminate among these disorders. It is of importance to distinguish PD from $\mathrm{AD}$ and $\mathrm{DLB}$, as the symptom profile, course and management of each disorder differs, Table 3 (Weintraub and Stern, 2005).

PD Dementia x Corticobasal Degeneration (CBD): In practice, this is one of the most frequent diagnostic confusions, especially when in the early stages of CBD. In CBD, besides significant cortical impairment, there is subcortical changes, but in PD dementia the latter is the only checked, causing therefore a standard type of subcortical dementia. The presence and importance of the tremor in the clinical setting of PD are much more prominent than in $\mathrm{CBD}$, where this signal occurs in just over half of cases (Kompoliti et al., 1998; Farsang et $a l ., 2005)$. The progression of the disease is faster in PD dementia than in CBD. One of the main features differentiating the two entities is the poor and not sustained response to levodopa in the case of CBD, contrary to what happens with PD (Koller and Montegomery, 1997; Pahwa and Lyons, 2010).
PD Dementia x Multiple System Atrophy (MSA): MSA is a complex neurodegenerative disorder characterized by autonomic dysfunction, Parkinsonism, cerebellar ataxia and pyramidal signs in any combination. Both MSA and PD are currently classified as alpha-synucleinopathies and both share some parkinsonian signs, but with different intensity, location and outcome, as well as different is the distribution of brain lesions in each disorder (Wenning et al., 2003). Despite some common elements, there are several differences between the two entities: MSA is a rare disease; MSA age of onset is earlier; outcome progresses faster in MSA; parkinsonism is less asymmetrical in MSA; tremor at rest is rare in MSA and frequent in PD; postural stability is compromised early in MSA unlike PD; hallucinations are practically inexistent in MSA, but occur with relative frequency in PD dementia; pyramidal and cerebellar signs occur in MSA, but not in the PD dementia; neuropsychological changes are milder (or even absent in the early stages) and the presence of dementia speaks against the possibility of MSA; symptoms such as orofacial dystonia, stridor and REM sleep behavior disorder are more common in MSA; poor response to levodopa in MSA; presence of oligodendroglial inclusions in neuropathology of MSA, but not in PD dementia (Wenning et al., 2004).

PD Dementia x Vascular Parkinsonism: In patients with vascular parkinsonism (usually due to subcortical arteriosclerotic encephalopathy, also known as Binswanger's disease) the extrapyramidal syndrome is dominated by bradykinesia and usually does not occur tremor at rest (and rarely there is stiffness, signs often found in PD dementia). Instead of these signs, it will be commonly observed in vascular parkinsonism: 
pseudobulbar palsy, gait disturbance and urinary incontinence, associated with dementia. Therapy with levodopa is ineffective, unlike what happens with PD. Moreover, in vascular parkinsonism there is no neuronal loss in the substantia nigra, but leucoaraiosis and lacunes in the brain white matter and in the basal ganglia (Szirmai and Kovacs, 2002).

PD dementia $x$ dementia in Normal Pressure Hydrocephalus (NPH): NPH is classically conceived as a ventricular enlargement without hypertension (average fluid pressure below $18 \mathrm{~cm}$ of water), accompanied by a progressive triad of symptoms: disturbance of gait, urinary incontinence and cognitive deterioration (Vanneste, 2000). If on the one hand a symptom triad of NPH can easily be found in PD dementia, on the other there are many anecdotal reports of NPH causing parkinsonism. While someone could argue that these patients were carriers of PD and NPH, the reversibility of extrapyramidal symptoms with CSF drainage and the lack of response to levodopa speak against this comorbidity (Krauss et al., 1997; Factora and Luciano, 2008). The differential diagnosis between these two entities, however, offers no great difficulty, since neuroimaging always show an exuberant dilatation of ventricles in NPH cases, an absent feature in cases of PD dementia.

Neuroimaging: A review of the initial findings of neuroimaging studies in patients with parkinsonism and dementia may be observed in a recent article (Burn and O'Brien, 2003). Several new studies of structural and functional neuroimaging expanded these findings. In general, structural neuroimaging of these patients will demonstrate a predominantly subcortical atrophy. The lateral ventricles (mainly the frontal horns), are often increased, which suggests, indirectly, this pattern of subcortical atrophy. Structural changes in the caudate, as assessed in vivo by MRI, do not appear to contribute to cognitive deterioration observed among patients with $\mathrm{PD}, \mathrm{PD}$ with dementia and AD. This finding was noted by Almeida et al. (2005) in their study of structural imaging using MRI and volumetry. According to them, there were no significant differences in the total volume of the brain or the caudate between PD and PD with dementia, while those with $\mathrm{AD}$ showed a significant decrease of the total brain and caudate volumes, compared to controls and patients with PD. There were no significant correlations between the volume of the caudate and overall cognitive function, executive performance or speed of processing.

In another study of magnetic resonance imaging, but functional (fMRI), using images related to a specific function, it was suggested that the hypoactivity in fronto-striatal circuits may underlie cognitive deficits in patients with early PD (Lewis et al., 2003). For this study, were compared groups of patients with PD, with and without cognitive deterioration, matched by all other clinical measures. Functional MRI showed significant reductions in the intensity of the signal during a paradigm of operative memory, in specific locations in frontal and striatal regions in individuals with cognitive deterioration, compared to those without cognitive impairment, suggesting that cognitive deficits in PD are accompanied by neural changes related to the modifications that accompany motor deficits, but are distinct from them.

A study with Single Photon Emission Tomography (SPECT), which measured the regional cerebral flow in patients with PD without dementia, demonstrated bilateral occipital and posterior parietal hypoperfusion, compared to controls (Abe et al., 2003). There was a strong positive correlation related to performance in a visual test (colored progressive matrices of Raven) and regional cerebral flow in the right visual association area, suggesting that the occipital hypoperfusion probably has relationship with the worsening of visual cognition in patients with PD.

Cholinergic deficits were described in pathological specimens of patients with PD and dementia. Cholinergic deficits in these patients may also be observed in vivo, using functional neuroimaging Positrons Emission Tomography (PET) and seems to be more serious than that seen in $\mathrm{AD}$ (Bohnen et al., 2003). The same study revealed that the mean activity of cortical acetylcholinesterase, compared with the controls, was lower in patients with PD and dementia (20\%), followed by those with PD without dementia (13\%) and $\mathrm{AD}(9 \%)$. The reduced activity of cortical acetylcholinesterase seems, then, be more characteristic of individuals with PD and dementia than those with mild AD.

Another study with PET, using 18F-dopa as a marker to assess the dopaminergic function in PD patients with dementia showed decreased capture of $18 \mathrm{~F}$-dopa bilaterally in the striatum, mesencephalon and anterior cingulate area, compared to normal controls (Ito et al., 2002). A relative difference in the uptake of 18F-dopa between PD and PD patients with dementia was a bilateral decline in anterior cingulate and ventral striatum and the right caudate nucleus, in the group of PD with dementia. The authors concluded that PD dementia is associated with a worsening of mesolimbic and caudate dopaminergic function. 
Treatment: PD survival after the onset of the disease is on average 9-12 years. These data of the natural history of PD, as previously exposed, give us some hints about the pharmacological approach in this disease: we will deal with predominantly elderly patients and that the medication should be used often for more than a decade. The treatment of dementia in idiopathic Parkinson's disease is even more complex, as it involves the joint treatment of two degenerative diseases, in theory, or at least the same degenerative process with two different phenotypic presentations (though physiopathologically should be probably related). The treatment of each condition separately is complex and therefore it is natural expect even greater complexity in simultaneous treatment of both conditions. The only FDA-approved medication for dementia associated with Parkinson's disease is rivastigmine, a cholinesterase inhibitor (Marder, 2010). The complexity in treating this combination increases even more when one remember that the treatment of a condition may interfere with the other. As an example of this, it may be recalled that the anticholinergic treatment usually used on PD may worsen the cognitive symptoms of dementia. Similarly, antipsychotic drugs used to treat changed behaviors in dementia may worsen extrapyramidal symptoms of PD. Dopaminergic drugs used in PD can create or aggravate behavioral symptoms associated with dementia and so on.

The cholinesterase inhibitors were tested in this population of patients on the basis of substantial deficits in cholinergic markers in PD with dementia. Preliminary evidence was gathered regarding the cholinesterase inhibitors as an effective treatment of dementia in PD, but they need to be confirmed by randomized controlled clinical trials that are sufficiently broad. These studies are in progress. Anyway, the usefulness of this group of drugs for dementia in PD seems to be clear and maybe for this are already widely used in medical practice. The question remains whether the anticholinesterasic effects of these medications could exacerbate some of the symptoms of PD (tremor, for instance) (Emre et al., 2007).

A study conducted by the higher current authority in dementia associated with PD and that became the reference in this area, provided reasonable evidence as to the usefulness of anticholinesterasics in the treatment of this condition (Giladi et al., 2003). In this randomized and placebo-controlled study, Emre et al. (2004) used rivastigmine in 541 patients (only 410 completed the study). A significant benefit was observed in the primary measures (Clinical Global Impression-CGI and ADAScog), but also in secondary measures (activities of daily living, neuropsychiatric
inventory-NPI, mini-mental state examination, verbal fluency tests, clock drawing and attention tests). Although was not observed worsening in motor symptoms in formal scales of measurement, an increase of the tremor was recorded among the side effects in some patients treated with rivastigmine. Among other side effects, were also reported high rates of nausea and vomiting?

In an open study, 28 patients with PD dementia were treated with rivastigmine for 26 weeks, with 20 of them completed the study (Fogelson et al., 2003). There was significant improvement in cognitive measures, including the mental subscore of Unified Scale for Assessment of Parkinson's Disease and in total score of cognitive subscale of the Alzheimer's Disease Assessment Scale (ADAS-Cog) at 26th week. The subscores in items of recognition, meeting of words, memorization of instructions and concentration from ADAS-Cog also improved significantly; the motor score of the Unified Scale for Assessment of Parkinson's disease remained unchanged. The same group described the effects of rivastigmine in quantitative electroencephalogram of patients with dementia and PD, which showed a significant increase in on alpha activity and a trend of increase in beta activity (Aarsland et al., 2003c). In another open study, 16 patients with PD dementia were treated with galantamine for eight weeks; 13 of them completed the study. It was verified improvement in overall mental score in eight patients, while four worsened. There was a significant improvement in the TDR and a trend of improvement in the Mini-Mental State Examination and verbal fluency. Hallucinations improved in seven of nine patients. Parkinsonism improved in six individuals, but a slight worsening of tremor was observed in three (Leroi et al., 2004). In another randomized, doubleblind and placebo-controlled study, which involved 16 patients, donepezil improved significantly the subscales of memory. There was a trend of improvement in psychomotor speed and attention. There were no differences between the two groups in relation to psychiatric status, motor function or activities of daily living at the beginning or end of the study. Four patients who took donepezil left early the research because of adverse events, two because of cholinergic side effects and one due to an increase in parkinsonism. The reason why the fourth case dropout was not mentioned (Burn and McKeith, 2003).

The results of these three studies, although limited by the open design and small sample, are aligned with those of previous clinical studies with donepezil, rivastigmine and tacrine, which were reviewed in a recent study and suggested that the cholinesterase 
inhibitors could be useful in the treatment of patients with dementia in PD (Fernandez et al., 2003). An open label study about the efficacy of donepezil in PDD was demonstrated that the cholinesterase inhibitor donepezil can improve cognitive and psychiatric deficits in DLB and PDD patients and this is reflected in a reduction in carer distress (Thomas et al., 2005).

One of the main clinical features of dementia in general and dementia in PD in particular is the presence of psychological and behavioral symptoms, especially psychotic symptoms. It is believed that the most effective medication to treat such symptoms in dementia associated with PD would be clozapine; however, by its difficulty in handling, in practice it is rarely used. A retrospective analysis of all PD patients who received quetiapine, an atypical antipsychotic, was made in a single center (Emre et al., 2004). Among the 106 patients with average duration of treatment of 15 months and an average dose of $60 \mathrm{mg} / \mathrm{day}, 82 \%$ had complete or partial resolution of psychosis, $18 \%$ had no improvement and $32 \%$ had motor worsening, but not sufficiently severe to lead to discontinuation. It is interesting to notice that dementia was found in those less responsive to quetiapine and motor worsening tended to occur more in demented patients. Intuitively, at present, since the studies in this area grow very soon and our conduct is based more on evidence, as a general rule should be given preference for atypical antipsychotics for the treatment of these disorders in dementia associated with PD.

If visual hallucinations, delusions, or agitation develop, anticholergic medication should be stopped, dopamine agonists should be reduced stepwise and levodopa treatment should be optimized. If required, treatment with atypical neuroleptics such as quetiapine or clozapine should be administered. For clozapine, patients require monitoring for possible side effects.

\section{CONCLUSION}

Considerable progress has been made in defining the scope of cognitive impairment in PD. Neuropsychological, imaging and pathologic studies suggest a common denominator referable to early dopamine deficiency but highlight an increasingly important focus on non-dopaminergic e.g., cholinergic pathway involvement. Although specific etiologies for cognitive impairment remain unknown, a symptomatic treatment has been approved and strategies for early intervention are envisioned.

Retrospective as well as prospective clinicalpathological studies reveal that dementia in PD best correlates with LB pathology, so that dementia in PD can be designated as a LB-associated dementia. Diagnosis of dementia must be based on the presence of deficits in at least two of the four core cognitive domains (attention, memory, executive and visuo-spatial functions) as shown in clinical and cognitive examination and be severe enough to affect normal functioning. Although there are some differences in the extent and profile of deficits in individiual cognitive domains compared to patients with $\mathrm{AD}$ (more prominent memory impairment in $\mathrm{AD}$, more prominent executive dysfunction in PDD), these may vary from patient to patient and cannot be used as the sole basis of diagnosis.

Imaging studies demonstrate atrophy and hypometabolism, more prominent in the temporal and posterior areas. However, there is no single ancillary investigation which would help to diagnose individual patients. Patients with Parkinson's disease should undergo early screening for signs of dementia so that further diagnostic and therapeutic steps can be taken in timely fashion, as indicated. Current treatment for PDD is symptomatic, modest and only transiently effective. Larger scale, placebo controlled clinical trials are needed to be explored in future studies to provide an evidence base to guide the management in PDD.

\section{REFERENCES}

Aarsland, D. and M.W.J. Kurz, 2010. The epidemiology of dementia associated with Parkinson disease. J. Neurol. Sci., 15: 18-22. DOI: 10.1016/j.jns.2009.08.034

Aarsland, D., C.G. Ballard and G. Halliday, 2004. Are Parkinson's disease with dementia and dementia with Lewy bodies the same entity? J. Geriatr. Psychiatry Neurol., 17: 137-145. DOI: 10.1177/0891988704267470

Aarsland, D., I. Litvan and D. Salmon, 2003b. Performance on the dementia rating scale in Parkinson's disease with dementia and dementia with Lewy bodies: Comparison with progressive supranuclear palsy and Alzheimer's disease. J. Neurol. Neurosurg. Psychiatry, 74: 1215-1220. DOI: 10.1136/jnnp.74.9.1215

Aarsland, D., J. Zaccai and C. Brayne, 2005. A systematic review of prevalence studies of dementia in Parkinson's disease. Mov. Disord., 20: 1255-1263. DOI: 10.1002/mds.20527

Aarsland, D., J.P. Larsen and N.G. Lim, 1999. Range of neuropsychiatric disturbances in patients with Parkinson's disease. J. Neurol. Neurosurg. Psychiatry, 67: 492-496. DOI: 10.1136/jnnp.67.4.492 
Aarsland, D., K. Andersen and J.P. Larsen, 2003a. Prevalence and characteristics of dementia in Parkinson disease: An 8-year prospective study. Arch. Neurol., 60: 387-392. DOI: 10.1001/archneur.60.3.387

Aarsland, D., L. Marsh and A. Schrag, 2009. Neuropsychiatric symptoms in Parkinson's disease. Mov Disord., 24: 2175-2186. DOI: 10.1002/mds.22589

Aarsland, D., M. Hutchinson and J.P. Larsen, 2003c. Cognitive, psychiatric and motor response to galantamine in Parkinson's disease with dementia. Int. J. Geriatr. Psychiatry, 18: 937-941. DOI: 10.1002/gps.949

Abe, Y., T. Kachi and T. Kato, 2003. Occipital hypoperfusion in Parkinson's disease without dementia: correlation to impaired cortical visual processing. J. Neurol. Neurosurg. Psychiatry, 74: 419-422. DOI: 10.1136/jnnp.74.4.419

Almeida, O.P., E.J. Burton and I. McKeith, 2003. MRI study of caudate nucleus volume in Parkinson's disease with and without dementia with Lewy bodies and Alzheimer's disease. Dement. Geriatr. Cogn. Disord., 16: 57-63. DOI: 10.1159/000070676

Behrmann, M., J. Grafman and F. Boller, 2001. Handbook of Neuropsychology. 2nd Edn., Elsevier Science Ltd, Amsterdam, ISBN-10: 0444503609 pp: 264.

Boeve, B.F., M.H. Silber and J.E. Parisi, 2003. Synucleinopathy pathology and REM sleep behavior disorder plus dementia or parkinsonism. Neurology, 61: 40-45. PMID: 12847154

Bohnen, N.I., D.I. Kaufer and L.S. Ivanco, 2003. Cortical cholinergic function is more severely affected in parkinsonian dementia than in Alzheimer disease: An in vivo positron emission tomographic study. Arch. Neurol., 60: 1745-1748. DOI: 10.1001/archneur.60.12.1745

Braak, H., K. Del Tredici and U. Rub, 2003. Staging of brain pathology related to sporadic Parkinson's disease. Neurobiol. Aging, 24: 197-211. DOI: 10.1016/S0197-4580(02)00065-9

Burn, D.J. and I.G. McKeith, 2003. Current treatment of dementia with Lewy bodies and dementia associated with Parkinson's disease. Mov. Disord., 18: S72-S79. DOI: 10.1002/mds. 10566

Burn, D.J. and J.T. O'Brien, 2003. Use of functional imaging in Parkinsonism and dementia. Mov. Disord., 18: S88-S95. DOI: 10.1002/mds.10568

Burn, D.J., 2006. Cortical Lewy bsody disease and Parkinson's disease dementia. Curr. Opin. Neurol., 19: 572-579. DOI: 10.1097/01.wco.0000247607.34697.a2
Cahn-Weiner, D.A., K. Williams and J. Grace, 2003. Discrimination of dementia with Lewy bodies from Alzheimer disease and Parkinson disease using the clock drawing test. Cogn. Behav. Neurol., 16: 8592. DOI: 10.1097/00146965-200306000-00001

Crucian, G.P., A.M. Barrett and D.W. Burks, 2003. Mental object rotation in Parkinson's disease. J. Int. Neuropsychol. Soc., 9: 1078-1087. PMID: 14738288

Emre, M., 2003a. Dementia associated with Parkinson's disease. Lancet Neurol., 2: 229-237. DOI: 10.1016/S1474-4422(03)00351-X

Emre, M., 2003b. What causes mental dysfunction in Parkinson's disease? Mov. Disord., 18: 63-71. DOI: $10.1002 / \mathrm{mds} .10565$

Emre, M., 2004. Dementia in Parkinson's disease: etiology and treatment. Curr. Opin. Neurol., 17: 399-404.

DOI: 10.1097/01.wco.0000137529.30750.ab

Emre, M., D. Aarsland, A. Albanese, E.J. Byrne and G. Deuschl et al., 2004. Rivastigmine for dementia associated with Parkinson's disease. N. Eng. J. Med., 351: 2509-2518. DOI: 10.1056/NEJMoa041470

Emre, M., D. Aarsland, R. Brown, D.J. Burn and C. Duyckaerts et al., 2007. Clinical diagnostic criteria for dementia associated with Parkinson's disease. Mov. Disord., 22: 1689-1707. DOI: 10.1002/mds. 21507

Factora, R. and M. Luciano, 2008. When to consider normal pressure hydrocephalus in the patient with gait disturbance. Geriatrics, 63: 32-37. PMID: 18312021

Farsang, M., Takats, A., Szirmai, I. and Kovacs, T., 2005. Clinical features of corticobasal degeneration. Ideggyogy Sz., 58: 45-51. PMID: 15884398

Fernandez, H.H., M.E. Trieschmann and M.A. Burke, 2003. Long-term outcome of quetiapine use for psychosis among parkinsonian patients. Mov. Disord., 18: 510-514. DOI: 10.1002/mds.10374

Fogelson, N., E. Kogan and A.D. Korczyn, 2003. Effects of rivastigmine on the quantitative EEG in demented Parkinsonian patients. Acta Neurol. Scand., 107: 252-255. DOI: 10.1034/j.16000404.2003.00081.x

Foltynie, T., C.E.C. Brayne, T.W. Robbins and R.A. Barker, 2004. The cognitive ability of an incident cohort of Parkinson's patients in the UK. The CamPaIGN study. Brain, 127: 550-560. DOI: 10.1093/brain/awh067

Galpern, W.R. and A.E. Lang, 2006. Interface between tauopathies and synucleinopathies: A tale of two proteins. Ann. Neurol., 59: 449-458. DOI: 10.1002/ana.20819 
Gelb, D.J., E. Oliver and S. Gilman, 1999. Diagnostic criteria for Parkinson's disease. Arch. Neurol., 56: 33-39. DOI: 10.1001/archneur.56.1.33

Giladi, N., H. Shabtai and T. Gurevich, 2003. Rivastigmine (Exelon) for dementia in patients with Parkinson's disease. Acta Neurol. Scand., 108: $\quad 368-373$. DOI: $\quad 10.1034 / j .1600-$ 0404.2003.00211.x

Ito, K., A. Nagano-Saito and T. Kato, 2002. Striatal and extrastriatal dysfunction in Parkinson's disease with dementia: A 6-[18F]fluoro-L-dopa PET study. Brain, 125: 1358-1365. DOI: 10.1093/brain/awf134

Kehagia, A.A., R.A. Barker and T.W. Robbins, 2010. Neuropsychological and clinical heterogeneity of cognitive impairment and dementia in patients with Parkinson's disease. Lancet Neurol., 9: 1200-1213. DOI: $10.1016 / \mathrm{S} 1474-4422(10) 70212-\mathrm{X}$

Kensinger, E.A., D.K. Shearer and J.J. Locascio, 2003. Working memory in mild Alzheimer's disease and early Parkinson's disease. Neuropsychology, 17: 203-239. PMID: 12803428

Koller, W.C. and E.B. Montgomery, 1997. Issues in the early diagnosis of Parkinson's disease. Neurology, 49: S10-25. PMID: 9222271

Kompoliti, K., C.G. Goetz and B.F. Boeve, 1998. Clinical presentation and pharmacological therapy in corticobasal degeneration. Arch. Neurol., 55: 957-961. DOI: 10.1001/archneur.55.7.957

Kovari, E., G. Gold and F.R. Herrmann, 2003. Lewy body densities in the entorhinal and anterior cingulate cortex predict cognitive deficits in Parkinson's disease. Acta Neuropathol., 106: 8388. PMID: 12687392

Krauss, J.K., J.P. Regel, D.W. Droste, M. Orszagh and J.J. Borremans et al., 1997. Movement disorders in adult hydrocephalus. Mov. Disord., 12: 53-60. DOI: $10.1002 / \mathrm{mds} .870120110$

Lanska, D.J., 2010. Chapter 33: The history of movement disorders. Handb. Clin. Neurol., 95: 501-546. DOI: 10.1016/S0072-9752(08)02133-7

Leroi, I., J. Brandt and S.G. Reich, 2004. Randomized placebo-controlled trial of donepezil in cognitive impairment in Parkinson's disease. Int. J. Geriatr. Psychiatry, 19: 1-8. DOI: 10.1002/gps.993

Levy, G., 2007. The relationship of Parkinson disease with aging. Arch. Neurol., 64: 1242-1246. DOI: 10.1001/archneur.64.9.1242

Lewis, S.J.G., A. Dove and T.W. Robbins, 2003. Cognitive impairments in early Parkinson's disease are accompanied by reductions in activity in frontostriatal neural circuitry. J. Neurosci., 23: 6351-6356. PMID: 12867520
Lyketsos, C.G., N. Kozauer and P.V. Rabins, 2007. Psychiatric manifestations of neurologic disease: Where are we headed? Dialogues Clin. Neurosci., 9: 111-124. PMID:17726911

Martinez-Martin, P., C. Falup-Pecurariu, C. RodriguezBlazquez, M. Serrano-Dueaas and F.J. Carod Artal et al., 2011. Dementia associated with Parkinson's disease: Applying the movement disorder society task force criteria. Parkinsonism Relat. Disord., 17: 621-624. DOI: 10.1016/j.parkreldis.2011.05.017

McKeith, I., 2007. Dementia with Lewy bodies and Parkinson's disease with dementia: Where two worlds collide. Pract. Neurol., 7: 374-382. DOI: 10.1136/jnnp.2007.134163

McKeith, I., D.W. Dickson, J. Lowe, M. Emre and J.T. O'Brien et al., 2005. Diagnosis and management of dementia with Lewy bodies: Third report of the DLB Consortium. Neurology, 65: 1863-1872. DOI: 10.1212/01.wnl.0000187889.17253.b1

McKeith, I., J. Mintzer, D. Aarsland, D. Burn and H. Chiu et al., 2004. Dementia with Lewy bodies. Lancet Neurol., 3: 19-28. DOI: 10.1016/S14744422(03)00619-7

McNamara, P., R. Dursa, A. Brown and A. Lynch, 2003. Counterfactual cognitive deficit in persons with Parkinson's disease. J Neurol. Neurosurg. Psychiatry, $\quad 74$ : 1065-1070. DOI: 10.1136/jnnp.74.8.1065

Mollenhauer, B., H. Forstl, G. Deuschl, A. Storch and W. Oertel et al., 2010. Lewy body and parkinsonian dementia: Common, but often misdiagnosed conditions. Deuts. Arztebl. Int., 107: 684-691. PMID: 20963199

Noe, E., K. Marder and K.L. Bell, 2004. Comparison of dementia with Lewy bodies to Alzheimer's disease and Parkinson's disease with dementia. Mov. Disord., 19: 60-67. DOI: 10.1002/mds.10633

Pahwa, R. and K.E. Lyons, 2010. Early diagnosis of Parkinson's disease: Recommendations from diagnostic clinical guidelines. Am. J. Manage. Care., 16: S94-S99. PMID: 20297872

Peran, P., O. Rascol and J.F. Demonet, 2003. Deficit of verb generation in nondemented patients with Parkinson's disease. Mov. Disord., 18: 150-156. PMID: 12539207

Perry, E.K., L. Kilford and A.J. Lees, 2003. Increased Alzheimer pathology in Parkinson's disease related to antimuscarinic drugs. Ann. Neurol., 54: 235238. DOI: $10.1002 /$ ana. 10639

Poewe, W.H. and G.K. Wenning, 2006. The natural history of Parkinson's disease. J. Neurol., 42: S146S152. PMID: 17131223 
Shoulson, I., 1998. Mortality in DATATOP: A Multicenter trial in early Parkinson's disease. Ann. Neurol., 43: $318-325 . \quad$ DOI: 10.1002/ana.410430309

Szirmai, I. and T. Kovacs, 2002. Parkinson syndrome and cognitive disorders. Ideggyogy Sz, 55: 220225. PMID: 12201229

Teive, H.A., J.A. Zavala, F.M. Iwamoto, D. Sa and H. Carraro, Jr. et al., 2001. As contribuições de Charcot e de Marsden para o desenvolvimento dos distúrbios do movimento nos séculos XIX e XX. Arq. NeuroPsiquiatr., 59: 633-636. DOI: 10.1590/S0004-282X2001000400031

Thomas, A.J., D.J. Burn, E.N. Rowan, E. Littlewood, J. Newby et al., 2005. A comparison of the efficacy of donepezil in Parkinson's disease with dementia and dementia with Lewy bodies. Int. J. Geriatr. Psychiatry, 20: 938-944. DOI: 10.1002/gps.1381
Vanneste, J.A., 2000. Diagnosis and management of normal-pressure hydrocephalus. J. Neurol., 247: 514. DOI: $10.1007 / \mathrm{s} 004150050003$

Weintraub, D. and M.B. Stern, 2005. Psychiatric complications in Parkinson disease. Am. J. Geriatr. Psychiatry, 13: 844-851. PMID: 16223962

Wenning, G.K., C. Colosimo, F. Geser and W. Poewe, 2004. Multiple system atrophy. Lancet Neurol., 3: 93-103. DOI: 10.1016/S1474-4422(03)00662-8

Wenning, G.K., F. Geser, M. Stampfer-Kountchev and F. Tison, 2003. Multiple system atrophy: An update. Mov. Disord., 18: S34-S42. DOI: 10.1002/mds. 10561 